\section{DiÁNOIA}

Diánoia

ISSN: 0185-2450

dianoia@filosoficas.unam.mx

Universidad Nacional Autónoma de México

México

FERNÁNDEZ VARGAS, MIGUEL ÁNGEL

Cómo justificar el veritismo

Diánoia, vol. LVI, núm. 67, noviembre, 2011, pp. 155-176

Universidad Nacional Autónoma de México

Distrito Federal, México

Disponible en: http://www.redalyc.org/articulo.oa?id=58433540009

Cómo citar el artículo

- Número completo

- Más información del artículo

Página de la revista en redalyc.org

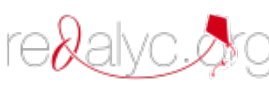

Sistema de Información Científica

Red de Revistas Científicas de América Latina, el Caribe, España y Portugal

Proyecto académico sin fines de lucro, desarrollado bajo la iniciativa de acceso abierto 


\title{
Cómo justificar el veritismo
}

\author{
Miguel Ángel FERnÁNDEZ VARGAS \\ Instituto de Investigaciones Filosóficas \\ Universidad Nacional Autónoma de México \\ mafv@filosoficas.unam.mx
}

\begin{abstract}
Resumen: Este trabajo esboza una forma de justificar el principio estructurador central de una teoría veritista de la evaluación epistémica, en respuesta a críticas planteadas por Eleonora Cresto a mi defensa del veritismo frente a una serie de objeciones en el sentido de que no es capaz de explicar la naturaleza y el valor del entendimiento. La primera sección presenta el esbozo de justificación del núcleo de una teoría veritista; la segunda responde a críticas más específicas de Cresto.
\end{abstract}

Palabras clave: valor epistémico, entendimiento, evaluación epistémica, creencia verdadera

\begin{abstract}
This paper sketches a way to justify the central tenet of a veritistic theory of epistemic evaluation, in response to problems raised by Eleonora Cresto concerning my defense of veritism, against a series of objetions to the effect that veritistic epistemology is uncapable of explaining the nature and value of understanding. The first section presents the sketch of justification of the nucleus of a veritistic theory, the second responds to some of Cresto's more specific criticisms.
\end{abstract}

Key words: epistemic value, understanding, epistemic evaluation, true belief

En el artículo “¿Son problemáticos para el veritismo la naturaleza y el valor del entendimiento?", ${ }^{1}$ discutí algunas objeciones recientes a la epistemología veritista en el sentido de que no puede dar cuenta de la naturaleza o el valor epistémico de una clase de entendimiento que denomino "entendimiento temático"; argumenté que las objeciones en cuestión no consiguen presentar ningún problema sustancial para el veritismo. En su artículo "Conocimiento y entendimiento: discusiones sobre el concepto de valor epistémico", 2 Eleonora Cresto ha planteado algunas preguntas acerca de las bases generales de mi defensa del veritismo y formulado críticas específicas relacionadas con algunos detalles de mis respuestas a las objeciones antiveritistas. En el presente artículo ofrezco el esbozo de una respuesta a las preguntas generales de Cresto

${ }^{1}$ M.Á. Fernández Vargas, “¿Son problemáticas para el veritismo la naturaleza y el valor del entendimiento?”, Diánoia, vol. 55, no. 65, noviembre de 2010, pp. 151173.

${ }^{2}$ Eleonora Cresto, "Conocimiento y entendimiento: discusiones sobre el concepto de valor epistémico", Diánoia, vol. 56, no. 66, mayo de 2011, pp. 165-177. 
y trato de responder a las críticas más puntuales que formula. Doy las gracias a Eleonora Cresto por darme la ocasión, con sus comentarios críticos, de aclarar y fundamentar mejor mi defensa del veritismo. ${ }^{3}$

\section{La formulación y la justificación del veritismo}

\subsection{La formulación del veritismo}

Mi defensa del veritismo ante las objeciones que considero en el artículo tiene como premisa una formulación específica de la que, pienso, es la tesis central, definitoria, de una teoría veritista de la evaluación epistémica:

(V) El valor de la creencia verdadera está esencialmente involucrado en la explicación de todo valor epistémico

Distingo entre una interpretación "dura" y una "tolerante" de (V). La interpretación "dura" concibe cualquier valor epistémico que sea distinto de la creencia verdadera como un valor meramente instrumental con respecto a la adquisición de creencias verdaderas. De modo que, según esta concepción, la manera como la creencia verdadera está involucrada en la explicación de cualquier valor epistémico es siempre la misma: como el valor intrínseco hacia el cual algo tiene que ser un buen medio si ha de ser epistémicamente valioso. En contraste, la lectura "tolerante" de $(V)$ permite que la creencia verdadera esté involucrada de diferentes maneras en la explicación de diferentes valores epistémicos. ${ }^{4}$ En el artículo uso como premisa una lectura tolerante de $(\mathrm{V})$ porque pienso que

\footnotetext{
${ }^{3}$ Me referiré a mi artículo con la letra "F" seguida del número de página, y al artículo de Cresto con la letra "C" seguida del número de página.

${ }^{4}$ La teoría veritista de Goldman 1999 parece seguir el espíritu del veritismo duro; Pritchard 2010, pp. 13-23, explícitamente discute el veritismo duro, y Brogaard 2009, pp. 284-285, lo presupone en su discusión. En general, es difícil documentar cuántos de los muchos epistemólogos que aceptan alguna versión del eslogan de que la verdad es la meta última de la cognición, o el valor epistémico cardinal, aceptarían la lectura dura de (V), porque la diferencia entre esa lectura y la tolerante muy rara vez se considera de manera explícita; de modo que en muchos casos no está claro con cuál querrían comprometerse los autores. No obstante, las teorías de algunos epistemólogos de hecho los comprometen a rechazar la lectura dura de (V) y a aceptar la lectura tolerante; éste es el caso, como lo señalo en F, pp. 153 y 170-171, de algunos epistemólogos de virtudes. Sin embargo, ni siquiera ellos consideran de manera explícita la lectura tolerante. Hasta donde yo sé, el único epistemólogo que explícitamente considera la lectura tolerante de (V) y que la desarrolla como una teoría general de la evaluación epistémica de la creencia es Alston 2005.
} 
sólo así es posible defender al veritismo de las objeciones basadas en los valores cognitivos asociados con el entendimiento: mientras que el veritismo duro parecería no poder explicar qué hay de epistémicamente valioso en diversos rasgos cognitivamente valiosos del entendimiento, e.g., la sistematicidad o el poder de unificación, simplemente porque no son conducentes a la verdad, el veritismo tolerante cuenta con mejores recursos para explicar en qué sentido esos rasgos pueden considerarse epistémicamente valiosos.

De modo que en el artículo queda claro, a mi parecer, que la lectura de (V) que uso como premisa en mi discusión es la lectura tolerante. ${ }^{5}$ Sin embargo, Cresto parece pensar que no es así, pues considera lo que piensa que son dos interpretaciones de la premisa que de hecho uso:

(LP) Todo valor epistémico diferente de la verdad es un medio para llegar a la verdad.

(LA) El valor epistémico total (de una creencia o de un conjunto de creencias) incluye necesariamente a la verdad; todo valor epistémico diferente de la verdad debe verse forzosamente como parcial, y como parte de un todo mayor. (C, p. 166)

De acuerdo con Cresto, (LP) es mi "lectura pretendida" de (V), pero por lo que he dicho debe ser claro que esto no puede ser así, pues mi lectura pretendida de (V) es tolerante, mientras que (LP) implica una lectura dura de (V). Por su parte, (LA) plantea otros problemas porque no está claro cómo interpretarla. Tomada al pie de la letra, (LA) parece ser equivalente a la afirmación de que la creencia verdadera es parte de cualquier valor epistémico total; Cresto parece apoyar esta intrepretación cuando glosa (LA) diciendo que "la verdad es parte fundamental de lo que entendemos por valor epistémico total" (C, p. 168). Pero es claro que esta tesis es falsa, pues todos, veritistas y antiveritistas, aceptan que hay valores epistémicos que no requieren la presencia de una creencia verdadera; por ejemplo, todos aceptan que puede haber creencias falsas justificadas, donde la justificación es algo epistémicamente valioso. (LA) debe entenderse de otra manera. Afortunadamente Cresto hace una glosa alternativa de (LA): "podemos estar de acuerdo en que todo valor epistémico incluye conceptualmente una referencia a la verdad, sin por ello pensar que todo valor epistémico incluye conceptualmente la verdad porque nos lleva a ella" (C, p. 167). Esto sí puede funcionar como la lectura de (V) que uso como premisa en el artículo, pues afirma que cualquier valor epistémico incluye una

${ }^{5}$ En F, p. 154, digo explícitamente que el veritismo que defenderé es el tolerante. 
referencia a la verdad, pero no dice que esa referencia sea siempre la misma; esa afirmación es compatible con que distintos valores epistémicos estén relacionados de diferente manera con la creencia verdadera $\mathrm{y}$, como he señalado, esta idea es la clave del veritismo tolerante.

Sin embargo, al plantear su principal pregunta acerca de mi formulación del veritismo, Cresto no tiene en mente la lectura tolerante de (V), sino que regresa a la lectura inaceptable (LP) y se pregunta cuál es la razón para aceptar la concepción de valor epistémico implícita en (LP):

(VE) Dícese o bien de la verdad, o bien de alguna característica de una creencia (teoría/conjunto de proposiciones) que nos conduce a la verdad. (C, p. 168)

Cresto nota que esta concepción del valor epistémico parece ser una mera estipulación del veritista y, por lo tanto, al presuponerla en su discusión contra el antiveritista, el veritista está trivializando el debate. Pues si (VE) es la concepción correcta de valor epistémico, entonces es trivial que los valores cognitivos del entendimiento que esgrime el antiveritista, y que no se ajustan a esa estipulación, serán correctamente rechazados por el veritista. Pero la pregunta no trivial es si esa concepción del valor epistémico es correcta.

Puesto que (LP) no es la tesis veritista que presupongo en el trabajo, podemos hacerla a un lado, junto con la cuestión de si la concepción asociada del valor epistémico (VE) es una mera estipulación. Sin embargo, podemos replantear las preguntas de Cresto y hacer que se refieran a la concepción del valor epistémico asociada con la lectura tolerante de (V), que sí presupongo en el trabajo. Para tener el contraste ante la mirada, podemos formular esa concepción de la siguiente manera:

(VE)* Dícese o bien de la creencia verdadera, o bien de alguna característica de una creencia (o conjunto de creencias) que es valiosa por ejemplificar algún tipo de relación adecuada con la verdad.

Las preguntas de Cresto serían entonces: ¿cuáles son las razones para aceptar la concepción del valor epistémico que (VE)* captura?, ¿cómo mostrar que (VE)* no es una mera estipulación? Cresto hace preguntas análogas a éstas en el contexto hipotético en el que un veritista desea rechazar que ciertas características de creencias sean epistémicamente valiosas sobre la base de que no encajan con su concepción de valor epistémico; en tal contexto ella afirma: "No parece buena idea [rechazarlas] simplemente por definición de valor epistémico. Necesitamos 
un argumento sustantivo $-\mathrm{y}$ la carga de la prueba la tiene aquí el veritista-" (C, p. 170). Cresto tiene razón en que para que mi defensa del veritismo sea plenamente satisfactoria es necesario ofrecer un "argumento sustantivo" a favor de (VE)*. La articulación detallada de un argumento tal es una cuestión muy compleja; en lo que resta de esta sección me limitaré a esbozar en términos muy generales la forma que, pienso, debe tomar el tipo de argumentación sustantiva que se requiere.

\subsection{La justificación del veritismo}

La meta de una teoría de la evaluación epistémica es estructurar una explicación acerca del valor de nuestros intentos por entrar en contacto cognitivo con el mundo, en cuanto tales. En vista del papel central de la creencia verdadera en la cognición, (VE)* es la propuesta básica del veritista para estructurar esa explicación. La tarea del veritista consiste en mostrar que su teoría puede dar cuenta exitosamente del campo de evaluación constituido por nuestros intentos por entrar en contacto cognitivo con el mundo. Planteadas así las cosas, está claro que el punto de partida del veritista no es una estipulación, sino una hipótesis que debe ponerse a prueba para determinar si puede dar cuenta del dominio para el cual se propone. A continuación esbozaré la manera como pienso que tendría que proceder el veritista para justificar la pretensión de que su hipótesis puede, en efecto, estructurar la evaluación del campo o dominio que le es propio.

Tal vez sea natural pensar que en $(\mathrm{VE})^{*}$ se mezclan dos ideas que habría que distinguir:

(1) La idea de que la creencia verdadera es el valor cardinal que está involucrado en la explicación de cualquier otro valor epistémico.

(2) La idea de que existen diversos tipos de relación adecuada con la verdad tales que una propiedad de creencias, o de conjuntos de creencias, tiene que ejemplificar al menos uno de esos tipos de relación con la verdad para poseer algún valor epistémico.

Sin embargo, tal como entiendo las cosas, la justificación de (1) no está desligada de la justificación de (2), pues considero que la manera más prometedora de justificar (1) supone justificar (2). Este proyecto de justificación debe comenzar con una muestra de diferentes juicios positivos acerca del valor epistémico de diversas creencias y conjuntos 
de creencias; enseguida deben identificarse las propiedades que fundamentan los juicios epistémicos considerados, es decir, las propiedades de las creencias en vista de las cuales éstas merecen la apreciación epistémica que los juicios expresan. Este procedimiento parece presentar al veritista una multiplicidad de propiedades epistémicamente valiosas; i.e., las múltiples propiedades de creencias que fundamentan los juicios epistémicos considerados. El desafío para el veritista es mostrar que el principio unificador de esa multiplicidad es que cada una de esas propiedades que fundamentan un juicio positivo de valor epistémico ejemplifica alguna relación adecuada con la verdad y, así, que la verdad está involucrada en la explicación de todas las propiedades epistémicamente valiosas que se hayan identificado en las creencias consideradas. De esta manera, la justificación de (1) depende de que el veritista consiga hacer plausible que las relaciones adecuadas con la verdad que él defina (llamémoslas relaciones- $V$ ) son capaces de dar cuenta de todas las propiedades de creencias (llamémoslas propiedades-E) que puedan fundamentar un juicio que atribuye valor epistémico a una creencia o conjunto de creencias. Es decir, la justificación de (1) depende de que se consiga hacer plausible que cualquier propiedad de creencias capaz de infundirles valor epistémico ejemplifica alguna de las relaciones con la verdad que el veritista haya definido. La idea es, pues, justificar el veritismo desplegando su poder explicativo. A continuación ilustraré con ejemplos concretos estas ideas esquemáticas y abstractas sobre el modo de justificar (VE)*.

Según he señalado, el teórico veritista tiene que comenzar compilando una muestra suficientemente variada de juicios que adscriben valor epistémico a creencias. ${ }^{6}$ Los juicios con los que deberá comenzar el veritista son aseveraciones como: " $S$ sabe que $p$ ", " $S$ tiene buenas razones para creer que $p$ ", "La evidencia de $S$ para creer que $p$ es só-

${ }^{6}(\mathrm{VE})^{*}$ define el valor epistémico como una propiedad de creencias; pero, como señalo en F, p. 169, una teoría veritista que sea comprensiva tiene que reconocer que hay otras unidades, distintas de las creencias, que pueden ser portadoras genuinas de valor epistémico, como los métodos, las prácticas y los rasgos de agentes. $(V E)^{*}$ podría generalizarse para cubrir todas estas unidades de evaluación; en consecuencia, la justificación de la tesis resultante sería más compleja que la justificación de $(\mathrm{VE})^{*}$ : habría que compilar juicios que atribuyan valor epistémico a métodos, prácticas y rasgos de agentes, desentrañar las propiedades de esas unidades que fundamentan los juicios que les atribuyen valor epistémico y describir relaciones con la verdad que tales propiedades ejemplifiquen, o deban ejemplificar, para ser capaces de infundir valor epistémico a las unidades que las posean. Por simplicidad, en este trabajo me concentro en la justificación de (VE)* que versa sólo sobre el valor epistémico de creencias.

Diánoia, vol. LVI, no. 67 (noviembre 2011). 
lida", etc. Las reflexiones que los epistemólogos han hecho sobre este tipo de juicios han dejado en claro que esos enunciados pueden usarse para expresar lo que de hecho son propiedades-E muy diferentes. El término "buenas razones" puede usarse para expresar una noción fundacionista o coherentista, "tener evidencias sólidas" puede expresar un fenómeno internista o uno externista, "saber" puede expresar un estado que implica justificación discursiva o uno que no la implica, etc., etc. La primera tarea del veritista es identificar las diversas propiedades-E de las creencias que pueden subyacer en los juicios ordinarios sobre valor epistémico. Afortunadamente, muchos epistemólogos se han encargado ya de hacer esa identificación. A continuación examinaré cinco propiedades-E de creencias que han sido profusamente discutidas:

(a) Estar basada en evidencia adecuada.

(b) Ser el resultado de una fuente fiable de formación de creencias.

(c) Ser el resultado del ejercicio de alguna competencia cognitiva propia.

(d) Haber sido formada sin violar ninguna obligación epistémica.

(e) Integrar un cuerpo de creencias coherente, sistemático y unificado que suministra explicación. ${ }^{7}$

Desde luego, podríamos hacer un catálogo más extenso de propiedadesE, pero para los propósitos ilustrativos que persigo en este trabajo las cinco anteriores serán suficientes. El siguiente paso en la estrategia justificatoria del veritista es formular un conjunto de relaciones-V que las creencias pueden mantener con la verdad, tales que cada una de las propiedades (a)-(e) sean valiosas en virtud de ejemplificar al menos una de esas relaciones-V. El valor de (a)-(e) así explicado será su valor distintivamente epistémico.

Con respecto a (a) y (b) la tarea del veritista es sencilla, pues está claro que la relación-V que ambas propiedades-E ejemplifican es:

(1) Tener una génesis que sea conducente a la verdad.

${ }^{7}$ Alston 2005, pp. 37-190, discute ampliamente éstas y otras propiedades-E o, como él las llama, "desiderata epistémicos", e intenta construir una explicación veritista del valor epistémico de esas propiedades. El tipo de justificación del veritismo que esbozo en el presente trabajo tiene algunos puntos de contacto con la teoría de Alston, pero también otros de sustancial desacuerdo. Dejo para otra ocasión la crítica de la teoría de Alston y la comparación de ella con el tipo de teoría que aquí esbozo. 
En efecto, si $B$ es el resultado de una fuente fiable de formación de creencias, o si ha sido formada con base en evidencia adecuada, de ello se sigue que la génesis de $B$ es conducente a la verdad. Sin embargo, hay complicaciones.

Por lo que respecta a (b), la fiabilidad de una fuente puede entenderse de diferentes maneras; en particular, las creencias que resultan de una fuente fiable pueden poseer al menos dos propiedades-E diferentes: ser una creencia sensible [sensitive] o ser una creencia segura [safe]. Puesto que la sensibilidad y la seguridad de las creencias se definen en términos de relaciones contrafácticas con la verdad, estas diversas propiedades-E asociadas con la propiedad (b) no representan ninguna dificultad para el veritista. ${ }^{8}$

Por lo que respecta a la propiedad (a), al sostener que ejemplifica (1) estoy asumiendo una lectura de "evidencia adecuada" según la cual si $E$ es evidencia adecuada de que $p, E$ es un elemento que hace objetivamente más probable que $p$. Algunos filósofos internistas rechazarían esta lectura de "evidencia adecuada" sobre la base de que existe la intuición de que un sujeto puede tener evidencia adecuada para creer que $p$ aun cuando sea sistemáticamente engañado por una supercomputadora que estimula artificalmente su cerebro. Un filósofo veritista puede reaccionar de diferentes maneras ante este tipo de posición, pero puesto que lo que me ocupa en este momento es ilustrar cómo justificar el veritismo desplegando el poder explicativo que puede tener, simplemente asumiré que la interpretación internista de (a) constituye un explanandum legítimo y, por lo tanto, que el veritista debe explicarlo. Si hemos asumido entonces que, bajo la interpretación internista, (a) constituye una genuina propiedad-E, ¿qué relación-V podría explicar su valor epistémico? Ya no puede ser (1), pues bajo la interpretación internista la propiedad (a) es epistémicamente valiosa, aunque tenerla de hecho no sea conducente a la verdad. Una hipótesis es que, bajo la interpretación internista de (a), ésta ejemplifica la siguiente relación-V:

(1)' Tener una génesis que desde el mejor punto de vista del sujeto parezca ser conducente a la verdad.

No entraré en los problemas teóricos que acarrea aceptar (1)', sólo la formulo como una opción viva para una epistemología veritista que desee respetar intuiciones internistas.

Pasemos a (c), ¿qué relación-V puede dar cuenta de su valor epistémico? Puesto que hay buenas razones para pensar que adquirir una

\footnotetext{
${ }^{8}$ Véase Pritchard 2008 para una discusión de las diferencias entre las nociones de seguridad y sensibilidad en cuanto propiedades-E de creencias.
} 
creencia mediante el ejercicio genuino de una competencia cognitiva propia no garantiza conductividad a la verdad, ${ }^{9}$ no podemos explicar el valor epistémico de (c) mediante (1) ni (1)'. Sin embargo, dado que generalmente los casos que se aducen como ejemplos en los que se presenta un ejercicio apto de competencias cognitivas sin conductividad a la verdad involucran algún tipo de anormalidad o carencia de optimalidad en las condiciones en las que se ejercitan las competencias, ${ }^{10}$ una conjetura verosímil es que la relación-V que explica el valor epistémico que se asocia a (c) es:

(2) Tener una génesis que sería conducente a la verdad si ciertas condiciones de normalidad u optimalidad se cumplieran. ${ }^{11}$

Pasemos a (d). Consideremos dos ejemplos de supuestas obligaciones epistémicas: "Reflexiona críticamente sobre las razones que se te ofrecen para creer algo", "Examina escrupulósamente la evidencia disponible". Generalmente este tipo de requerimientos se entienden de tal manera que un sujeto puede cumplir con ellos y, sin embargo, su creencia tener una genésis que de hecho no es conducente a la verdad; por lo tanto, la explicación del valor epistémico de (d) no puede ser que ejemplifique (1). La relación (1)' tampoco puede ser la explicación, pues es posible que un sujeto cumpla con sus obligaciones epistémicas y que, a pesar de ello, su perspectiva subjetiva sobre su situación siga siendo una desde la que le parece que la génesis de su creencia no es conducente a la verdad, por ejemplo, si cree falsamente que, en su situación, haber cumplido con esas obligaciones no lo acerca a la verdad. De modo que (d) puede no ejemplificar (1) y aun así ser una propiedad epistémicamente valiosa. Por lo que respecta a (2), suele asumirse que cuando un sujeto cumple con sus obligaciones epistémicas, las condiciones para la operación de sus facultades cognitivas son normales; por lo menos está

${ }^{9}$ Véase, por ejemplo, Sosa 2007, p. 31. Sin embargo, la relación entre el ejercicio de las competencias cognitivas propias y la conductividad a la verdad es un asunto complicado. Véase una discusión de tal complejidad, tal como se manifiesta en la epistemología de virtudes de Sosa, en Fernández 2010b.

${ }^{10}$ Véase, por ejemplo, Sosa 2007, pp. 30-33.

${ }^{11}$ Ésta no es, al menos explícitamente, la razón por la que los epistemólogos de virtudes consideran que la propiedad (c) sea epistémicamente valiosa. Su explicación es más bien que (c) es epistémicamente valiosa porque hace atribuible a $S$, como un logro propio, un éxito que es de naturaleza epistémica, i.e., una creencia verdadera (véanse, por ejemplo, Sosa 2003, pp. 173-175, y Greco 2003). Pero esta explicación no puede ofrecerse cuando la creencia formada competentemente resulta ser falsa. El tipo de veritismo que estoy esbozando puede dar cuenta del valor epistémico de (c) aun en esos casos. 
claro que satisfacer tales obligaciones no excluye tal normalidad. Por lo tanto, no parece que cumplir esas obligaciones sería un método conducente a la verdad si se satisficieran condiciones de normalidad, pues tales condiciones pueden de hecho estar satisfechas y el cumplimiento de tales obligaciones no ser conducente a la verdad; es decir, (d) no ejemplifica (3).

Necesitamos una nueva relación- $V$ que pueda explicar el valor epistémico de (d); una relación que podría realizar este trabajo es la siguiente:

(3) Ser el mejor intento intersubjetivamente reconocido de llegar a creencias verdaderas.

Tal como generalmente se entiende (d), las obligaciones epistémicas que se aplican a un sujeto no deben exceder el límite superior de lo que razonablemente puede requerirse de él, en vista de la meta de formar creencias verdaderas. Fallar en el cumplimiento de un requerimiento tal constituye una falta culposa, criticable; pero una falta con respecto a un requerimiento que va más allá de lo que razonablemente puede exigírsele no constituye una falta culposa, criticable. Puesto que el sujeto puede tener una concepción errónea sobre el límite superior de lo que razonablemente puede requerirse de él y, por lo tanto, de las obligaciones que se aplican a él, el umbral de lo que puede razonablemente requerirse de él y de qué obligaciones se le aplican se fija desde un punto de vista intersubjetivo. Éste es un esbozo de justificación de la tesis de que la propiedad (d) ejemplifica (3). ${ }^{12}$

Pasemos finalmente a (e). La propiedad (e) no es una característica de creencias individuales, sino de sistemas de creencias; en particular, se trata de una propiedad compleja que incluye coherencia, sistematicidad y unificación. ¿Qué relación con la verdad podría dotar de un valor distintivo a este ensamblaje de propiedades? Una de las objeciones clásicas a las teorías coherentistas de la justificación epistémica

${ }^{12}$ La cuestión del papel que desempeñan consideraciones intersubjetivas en la determinación del umbral de lo que es razonable requerir epistémicamente de un sujeto, y de qué obligaciones epistémicas se le aplican, rara vez se discute de manera explícita en los trabajos sobre obligaciones epistémicas. El foco de la discusión ha sido más bien si la aplicación del concepto de obligación en la evaluación epistémica acarrea compromisos insostenibles, como el llamado "voluntarismo dóxico" (véanse Alston 1988, y Feldman 1988). Sin embargo, me parece que una presuposición velada en esas discusiones es que $s i$ hay obligaciones epistémicas, entonces habrá consideraciones intersubjetivas que desempeñen un papel preponderante en la determinación de su aplicabilidad.

Diánoia, vol. LVI, no. 67 (noviembre 2011). 
es que el tipo de coherencia que se describe en (e) puede estar totalmente desligado de la verdad. Los delirios de un demente pueden ser coherentes, sistemáticos, estar unificados por principios generales y suministrarle al enfermo explicaciones de muchos fenómenos, pero claramente constituyen un intento estéril por entrar en buen contacto cognitivo con el mundo y, por lo tanto, tienen nulo valor epistémico. Esto sugiere que la propiedad (e) per se no infunde valor epistémico a los sistemas de creencias que la poseen, sino que para que su presencia pueda infundir tal valor es necesario que las creencias individuales que conforman el sistema en cuestión estén libres de los defectos que infectan a las creencias del demente; es decir, deben poseer ellas mismas algún valor epistémico. De modo que podría proponerse la hipótesis de que para que la propiedad (e) infunda algún valor epistémico tiene que ejemplificar la siguiente relación-V:

(4) Las creencias individuales así sistematizadas poseen algún valor epistémico.

Si las creencias individuales sistematizadas no poseyeran algún valor epistémico, digamos que si no fueran verdaderas y además no tuvieran ninguna de las propiedades (a)-(d), no es para nada claro por qué deberíamos considerar que un sistema de creencias que ejemplifique (e) posea valor epistémico. Imagínese que estamos evaluando un sistema de creencias que es coherente, que es rico en sus descripciones de fenómenos diversos, que unifica mediante hipótesis generales muchas de tales descripciones, que se apoya sólidamente en evidencia recabada y que ofrece explicaciones sistemáticas de los fenómenos que describe. Pero supóngase que las descripciones de los fenómenos en cuestión son en su mayor parte falsas, al igual que las hipótesis unificadoras y las descripciones de las evidencias recabadas; los principios generales del sistema unifican los fenómenos y encajan con las descripciones de la evidencia sólo porque todo es parte de una enorme y muy bien sincronizada y armónica distorsión de la realidad. Un sistema tal no ofrecería explicaciones genuinas de nada: sistematizar un grupo de hipótesis erróneas, junto con un conjunto de datos espurios, no constituye entendimiento de ningún fenómeno real. Si a la ausencia de verdad añadimos ausencia de las propiedades (a)-(d) en las creencias sistematizadas, se vuelve aún más claro que la propiedad (e) per se no engendra ningún valor epistémico. Ahora tenemos que imaginar un sistema de hipótesis generales, descripciones de fenómenos y descripciones de datos que, además de ser mayoritariamente falsas, no están apoyadas en evidencias adecuadas (ausencia de (a)), se ha llegado a ellas utilizando 
métodos y procedimientos que no son fiables (ausencia de (b)), violando toda clase de obligaciones y requerimientos epistémicos (ausencia de (d)) y que no son el resultado del ejercicio de competencias cognitivas relevantes (ausencia de (c)). Es patente que un sistema de creencias que tuviera todos estos defectos, por más coherente y sistematizado que fuera, no poseería valor epistémico alguno. Para que (e) sea una propiedad que conlleve algún valor epistémico debe ejemplificar (4).

Hasta aquí he ilustrado de manera esquemática el tipo de pasos cruciales en la justificación del veritismo: (i) identificar propiedades de creencias que infunden valor epistemico, i.e., las propiedades-E, y (ii) describir relaciones-V con la verdad que las propiedades-E deben ejemplificar para poseer algún valor epistémico. Puesto que el veritismo dice que todo valor epistémico se explica mediante una relación apropiada con la verdad, esto parecería implicar que la justificación plena de la teoría veritista se alcanzará sólo si se muestra que cualquier propiedad-E ejemplifica al menos alguna relación-V. Evidentemente, lo que he hecho hasta este momento no consigue esa meta, porque no he demostrado que las propiedades-E que he discutido sean todas las propiedades-E; de hecho, sabemos que no son todas. Cada teoría epistémica, cada epistemólogo, es capaz de definir nuevas propiedades-E. No tengo la menor idea de cómo demostrar a priori que cualquier propiedad de creencias que pueda definirse y que se engarce con alguna intuición que haga plausible juzgarla como epistémicamente valiosa, sea tal en virtud de ejemplificar una relación-V. Esto nos lleva de regreso a lo que señalé al inicio de esta sección: el veritista tiene que reconocer que (VE)* es una hipótesis para estructurar una teoría acerca del valor epistémico de nuestros intentos por entrar en contacto cognitivo con el mundo, y como tal se encuentra permanentemente a prueba. La justificación para aceptar la hipótesis veritista crece gradualmente a medida que el teórico veritista muestra cómo el valor de nuevas propiedades-E puede explicarse subsumiéndolas bajo alguna relación-V. Éste es un caso especial del fenómeno epistémico en el que la aceptabilidad de una hipótesis crece al expandir su poder explicativo.

Desde luego que en esta tarea de corroboración habrá casos difíciles para el veritista; pero cuál deba ser la reacción ante casos tales tampoco puede determinarse a priori. Por ejemplo, si el veritista encuentra alguna propiedad-E recalcitrante, en el sentido de que parece ser valiosa en relación con nuestros intentos por entrar en contacto cognitivo con el mundo, pero ese valor se resiste a ser subsumido bajo alguna relación-V, la decisión teórica entre rechazar los juicios de valor epistémico que se 
fundan en esa propiedad-E o modificar el cuerpo de la teoría veritista para dar cuenta de ella es algo que el veritista tendrá que determinar con base en consideraciones holistas. Estas consideraciones estarán guiadas por el principio de que ni los juicios de valor epistémico que se fundan en esa propiedad-E, ni los principios veritistas que encuentran difícil acomodar ese juicio, tienen prioridad unos sobre otros. ${ }^{13}$ Tanto los juicios como los principios teóricos destinados a dar cuenta de ellos son revisables unos a la luz de los otros. Esta simetría epistémica entre los juicios iniciales que una teoría busca explicar y los principios teóricos de la explicación es un sello del método de equilibrio reflexivo, y un sello que la empresa de justificar el veritismo debe adoptar. ${ }^{14}$

\section{Respuesta a objeciones específicas}

En esta sección responderé a críticas y problemas más específicos planteados por Cresto.

\subsection{Conocer proposiciones frente a conocer relaciones ${ }^{15}$}

En el artículo discuto la caracterización que hace Kvanvig del entendimiento temático en términos de un "apreciar", "ver" o "aprehender" las relaciones explicativas entre elementos de un cuerpo de información. Mi intención era hacer ver que el uso de esa terminología cuasimeta-

${ }^{13}$ Dadas las dimensiones de este trabajo, no puedo ofrecer aquí ilustraciones del tipo de estrategia que en el texto principal describo en términos muy abstractos y que, pienso, el veritista debe perseguir al encontrar casos difíciles de valores epistémicos que parecen resistirse a una explicación en términos veritistas. En mi discusión de las propiedades (a)-(e), supra, asumí que dichas propiedades son explananda legítimas para una teoría del valor epistémico, con el fin de ilustrar cómo el veritista puede subsumirlas bajo alguna relación-V apropiada. Pero esta actitud no tiene por qué ser la actitud del veritista ante cualquier aparente propiedad-E de creencias; en ocasiones, el veritista puede optar por rechazar que la aparente propiedad-E sea en realidad una propiedad que infunda valor epistémico. El tipo de argumentación que tiene que ofrecer para sustentar un rechazo tal puede ejemplificar también el tipo de argumentación compleja que tendría que guiar su juicio en casos recalcitrantes como aquellos a los que me refiero en el texto principal. Un ejemplo de ese tipo de argumentación puede verse en Alston 2005, pp. 5880 , donde rechaza que la aparente propiedad (d) que he discutido antes sea una genuina propiedad que infunda valor epistémico.

${ }^{14}$ Las formulaciones pioneras del método de equilibrio reflexivo se encuentran en Rawls 1971, pp. 42-43, y en Goodman 1965, pp. 63-64.

${ }^{15}$ Las siguientes tres subsecciones llevan los títulos que tienen las subsecciones correspondientes 3.A, 3.B y 3.C en el artículo de Cresto. 
fórica contribuye a crear un halo sui generis alrededor de la naturaleza del entendimiento, y que el examen de lo que se busca expresar mediante esos términos rápidamente revela que el fenómeno cognitivo en cuestión es mucho más asequible a un tratamiento veritista de lo que en un principio podría parecer.

En el artículo comienzo señalando una lectura natural de aquellos vocablos en la cual implican conocimiento; esta lectura no está disponible para Kvanvig, pues volvería incoherente su posición, ya que el objetivo de su argumentación es mostrar que el entendimiento no es una especie de conocimiento (2003, p. 188). Kvanvig basa su argumento en un caso en el que uno adquiere entendimiento consultando una fuente, digamos que un libro, que es correcta por accidente; sostiene que esa suerte epistémica impide el conocimiento de las proposiciones que integran el cuerpo de información que uno entiende, sin impedir el entendimiento. Señalo que, en su ejemplo, Kvanvig no considera de manera explícita otro conocimiento que un veritista podría pensar que constituye el entendimiento en cuestión: el conocimiento de las relaciones explicativas entre las proposiciones que integran el cuerpo de información. Puesto que, por mor de la coherencia, Kvanvig tampoco puede aceptar que el entendimiento consista en el conocimiento de esas relaciones, busco ser caritativo y completo el ejemplo de Kvanvig de tal manera que el conocimiento de las relaciones también sea minado por la suerte epistémica en cuestión, en virtud de que tal conocimiento se adquiere a través de la misma fuente que el conocimiento de las proposiciones. De esta forma se preserva el resultado que Kvanvig busca, que el conocimiento, sea de las proposiciones que componen el cuerpo de información, sea de las relaciones explicativas que se dan entre ellas, puede ser impedido por la suerte epistémica sin que esto impida el entendimiento. Mi conclusión, a partir de esto, es que la lectura más natural de los términos que Kvanvig usa para caracterizar el entendimiento en definitiva no le está disponible.

Cresto objeta que si la fuente del conocimiento de las relaciones explicativas fuera diferente de la fuente del conocimiento de las proposiciones relacionadas - por ejemplo, si conociéramos las relaciones por "reflexión cuidadosa" (C, p. 173), no por el libro-, entonces, en el ejemplo de Kvanvig, la suerte minaría el segundo conocimiento sin minar el primero y, entonces, podríamos tener conocimiento de las relaciones aunque no lo tuviéramos de las proposiciones relacionadas. Sin duda esto es cierto, pero esa posibilidad debilita el argumento de Kvanvig. Dado que su objetivo es argumentar que el entendimiento no es una especie de conocimiento, busqué interpretar su ejemplo de un 
modo tal que apoyara mejor su conclusión. Cresto imagina una modificación del ejemplo en la que queda abierta la posibilidad de identificar el entendimiento con un tipo de conocimiento, i.e., el de las relaciones explicativas, que no sería afectado por la suerte que impide el conocimiento de las proposiciones relacionadas. Esto pone en problemas a Kvanvig, pues él tendría que aderezar su ejemplo de tal forma que resultara verosímil que la fuente alternativa mediante la que uno aprende sobre las relaciones explicativas también pudiera estar afectada por suerte epistémica (y, por lo tanto, no entregaría conocimiento de tales relaciones) y que aun así tendríamos entendimiento. Tal vez el ejemplo de Kvanvig pueda modificarse exitosamente de esta manera, pero el hecho es que la modificación le complica el trabajo a él y se lo facilita al veritista. Éste acepta felizmente la modificación que imagina Cresto, pues deja la puerta abierta para poder identificar de manera verosímil el entendimiento con el conocimiento de las relaciones explicativas, lo que automáticamente lo hace manejable para el veritista.

Una segunda lectura que considero de los términos "ver" y "apreciar" relaciones explicativas es dóxica, según la cual ver o apreciar relaciones explicativas entre elementos de un cuerpo de información equivale a creer que ciertas relaciones explicativas se dan entre esos elementos. Sostengo que, para tener entendimiento, no basta con tener creencias que de hecho articulen correctamente las relaciones explicativas, sino que esas creencias tienen que estar justificadas, y lo ilustro con un ejemplo en el que uno tiene razones para dudar de esa articulación, por ello no está justificado en creer que esa articulación es correcta y por ello no tiene entendimiento. Cresto interpreta mi ejemplo al revés, pues señala que "no parece claro que la falta de justificación per se constituya una razón para dudar" (C, p. 173). Esto puede ser correcto, pero no afecta mi argumento, pues lo que yo digo es lo inverso: que si uno tiene una razón para dudar, entonces no está justificado. Otro aspecto en el que parece interpretar equivocadamente mi ejemplo se refiere al objeto de la duda. Señala que "no está claro que la duda sobre la verdad de $p_{1}, p_{2} \ldots p_{n}$ (ini siquiera la creencia en su falsedad!) nos impida conocer las relaciones relevantes entre ellas" (ibid.). Nuevamente, esto puede ser correcto pero no afecta a mi argumento, pues en mi ejemplo el objeto de la duda es la articulación de las relaciones explicativas, no la verdad de las proposiciones relacionadas.

\subsection{Rechazo de la lectura dóxica}

En el artículo discuto otra fuente del halo sui generis del entendimiento: el hecho de que a veces podemos obtenerlo mediante vehículos que aca- 
rrean información en formato no proposicional. Doy algunas razones para pensar que del hecho de que un estado de entendimiento se logre mediante un vehículo que no tiene estructura proposicional no se sigue que el estado mismo no tenga contenido proposicional. Cresto está de acuerdo con esto; sin embargo, señala que hay una manera "obvia" en la que el antiveritista puede llenar el vacío en su argumento. Consistiría simplemente en apelar a "consideraciones fenomenológicas: de hecho, en los casos típicos que el antiveritista tiene en mente, los sujetos no son conscientes de tener ninguna actitud especial frente a alguna proposición particular" (p. 174). Pero ésta es una premisa muy débil: las creencias no tienen que ser conscientes para ser reales; el ejemplo más patente de esto lo constituyen las innumerables creencias tácitas que en cualquier momento es correcto adscribir a cada uno de nosotros, ninguna de las cuales es una creencia de la que seamos conscientes en el momento de la adscripción. ${ }^{16}$

Pero Cresto piensa que puede ilustrar la verdad de la premisa que le ofrece al antiveritista con el siguiente ejemplo:

Imaginemos que $S$ es particularmente bueno para resolver el "juego del trabado" (un juego de ingenio popular entre los niños, que requiere mover piezas — cuadrados y rectángulos- de diferente tamaño en un tablero hasta conseguir liberar una de ellas). $S$ mueve las piezas muy rápido sin pensar exactamente qué es lo que está haciendo; sin embargo, en muy poco tiempo siempre llega a la solución. (C, p. 174)

Cresto pregunta si el entendimiento del juego que se demuestra en el desempeño competente en la búsqueda de la solución "¿... podría transformarse en proposicional?", y responde que "típicamente el jugador no efectúa de hecho dicha transformación" (C, p. 174).

Deseo señalar dos cuestiones con respecto a este ejemplo. En primer lugar, no está claro que el sujeto ejemplifique el tipo de entendimiento que tienen en mente los antiveritistas que considero. Zagzebski (2001, p. 241) y Riggs (2003, p. 217) piensan en el entendimiento de un área temática, de máquinas complejas, materias o disciplinas, pruebas matemáticas, en una palabra, en un cuerpo de información complejamente estructurado; esto no parece estar presente en el ejemplo de Cresto. Es verdad que Zagzebski sostiene que si uno ha conseguido entender, entonces sabe cómo hacer algo bien (loc. cit.), pero de esto no se sigue el

${ }^{16}$ Véase Lycan 1986 para una discusión de las creencias tácitas que deja claro cómo su existencia está disociada de la conciencia presente.

Diánoia, vol. LVI, no. 67 (noviembre 2011). 
condicional opuesto: que si uno sabe hacer algo bien, entonces ha conseguido el tipo de entendimiento que ella tiene en mente. Éste parece ser justamente el caso del ejemplo de Cresto: el sujeto sabe hacer algo muy bien, i.e., liberar las piezas geométricas requeridas; pero de esto no se sigue que tenga entendimiento de algo, en el sentido de dominar las relaciones explicativas entre los elementos de un cuerpo de información complejamente estructurado. Este tipo de entendimiento no parece desempeñar ningún papel en la explicación del desempeño del sujeto en el ejemplo de Cresto; su desempeño parece más bien ser un logro cognitivamente más rudimentario, una respuesta motriz coordinada directamente por los insumos de percepción acerca de las formas geométricas percibidas, tanto de las figuras sólidas como de los espacios vacíos disponibles.

En segundo lugar, podríamos aderezar el ejemplo de Cresto de tal forma que resultara verosímil que ejemplifique el tipo de entendimiento que los antiveritistas consideran. Por ejemplo, podríamos suponer que el sujeto aprendió a jugar mediante la guía de un maestro sumamente reflexivo que le suministró una lista de consejos explíctos para mejorar su desempeño, los cuales han sido "internalizados" a tal grado por el jugador que, al seguirlos, la rapidez de su desempeño no se ve afectada en absoluto. La pregunta aquí sería si el entendimiento que el sujeto ha logrado gracias a la asimilación de la instrucción explícita puede o no explicarse atribuyéndole estados dóxicos/proposicionales. Estoy abierto a aceptar que en un caso así pueda haber razones empíricas de peso que recomienden no adscribir creencias al sujeto como parte de la explicación de su desempeño, ${ }^{17}$ por ejemplo, razones acerca de cuáles son los mecanismos psicológicos que entran en operación durante ese

${ }^{17}$ Un característica del ejemplo de Cresto que parece sugerirle que el entendimiento del sujeto no puede explicarse en términos de creencias es que la rapidez con la que ocurre el desempeño competente del sujeto se da "sin pensar exactamente qué es lo que está haciendo". Creo que aquí hay un peligro de confundir rasgos del desempeño o la ejecución con rasgos del estado de entendimiento que puede cumplir algún papel en la explicación de ese desempeño o ejecución. La manera como ocurre el desempeño puede ser evidencia de que hay entendimiento, pero no es un rasgo constitutivo del entendimiento mismo: la manera como de hecho ocurre el desempeño puede variar en virtud de multitud de contingencias externas (una droga administrada, un contexto de rivales hostiles, etc.) sin que ello afecte para nada el entendimiento que el sujeto tiene del juego. Esta disociación entre desempeño y entendimiento sugiere, en particular, que el tipo de inferencia que Cresto desea hacer, de ciertos rasgos del desempeño a ciertos rasgos del estado de entendimiento, no es automática; requiere apoyarse en el tipo de razones empíricas que señalo en el texto, las cuales, a su vez, pueden ser discutibles.

Diánoia, vol. LVI, no. 67 (noviembre 2011). 
desempeño rápido y si éstos influyen en el desempeño mediante entregas que realicen a una "caja de creencias" o de alguna otra manera más directa. ${ }^{18}$ Pero Cresto no ofrece este tipo de razones; excluir a las creencias de tener un papel explicativo en un caso así simplemente porque el sujeto no es consciente de tenerlas durante su desempeño es, como ya dije, muy poco convincente.

Quiero enfatizar que la meta de mi crítica a los antiveritistas no era demostrar o establecer que todos los casos de entendimiento que consideran tienen una naturaleza dóxica y, por lo tanto, proposicional, sino simplemente hacer ver que las razones que ellos ofrecen en contra de esta hipótesis no son sólidas. Mi respuesta a la ayuda que Cresto les ofrece es exactamente del mismo estilo: tal vez el caso que ella describe represente alguna dificultad para el veritismo, pero la razón que ella ofrece para pensar que eso sea así no es sólida.

\section{3. Valores epistémicos}

Un tipo de objeción al veritismo que considero en el artículo se basa en identificar características del entendimiento que son valiosas de un modo que el veritismo no puede explicar. Una de esas características es la sistematicidad. Discuto la posición de Kvanvig, que presenta como un problema para el veritismo el hecho de que la sistematicidad en un cuerpo de creencias es valiosa de modos que no tienen que ver con la verdad: por ejemplo, por ser prácticamente útil y psicológicamente

${ }^{18}$ En el texto principal estoy asumiendo que en el caso descrito el entendimiento sí tiene un papel central en la explicación causal del desempeño. En vista de ello, si las razones empíricas recomendaran no adscribir estados dóxicos al sujeto como parte de la explicación causal de su desempeño, esto podría interpretarse como evidencia de que el entendimiento no consiste en estados dóxicos, lo cual sería problemático para el veritismo. Pero el veritista podría acomodar esas razones empíricas abandonando la suposición de que en el caso considerado el entendimiento tiene un papel central en la explicación causal del desempeño. Por ejemplo, una hipótesis sería que el entendimiento mismo que el sujeto ha logrado puede explicarse en términos de estados dóxicos, pero que, gracias a la práctica en la resolución de múltiples casos del juego, el papel causal de ese entendimiento gradualmente disminuye hasta que deja su lugar a habilidades más automáticas que, aunque se han desarrollado gracias a ese entendimiento, llegan a sustituirlo en la explicación causal del desempeño. Si esta hipótesis empírica fuera correcta, entonces, aun cuando fuera verdad que la explicación causal del desempeño del sujeto, en el ejemplo de Cresto que he aderezado, no requiriera la atribución de estados dóxicos al sujeto, ello no mostraría que el entendimiento mismo que el sujeto posee no consista en estados de tal naturaleza; sólo indicaría que el entendimiento que el sujeto logró ya no tiene en el presente un papel central en la explicación causal de su desempeño.

Diánoia, vol. LVI, no. 67 (noviembre 2011). 
agradable. Señalo que Kvanvig no da una razón para pensar que estos valores sean epistémicos, i.e., tales que una teoría del valor epistémico tendría que explicarlos, mientras que el veritista tiene una razón clara para excluirlos. Cresto insiste en que esa razón del veritista se basa en una mera definición estipulativa de valor epistémico (C, p. 175); pero ya expliqué en la primera parte de este trabajo que esto no es así. La hipótesis del veritista forma parte de una red teórica robustamente corroborada, en virtud de lo cual puede funcionar como premisa en la exclusión de la utilidad práctica y el placer psicológico del dominio de evaluación que es propio de la teoría del valor epistémico. El antiveritista tendría que dar un respaldo teórico similar a su pretensión de que esos valores, después de todo, son de incumbencia para una teoría del valor epistémico.

Cresto sostiene que cuando razono del modo anterior en contra del antiveritista oscilo entre defenderme de dos objeciones diferentes que se le pueden hacer al veritismo:

(7) El veritismo no da cuenta de $x$, pero debería, porque es parte de lo que se le pide a una teoría epistémica.

(8) El veritismo no da cuenta de $x$, y está bien que no lo haga, pero eso muestra que hay otros tipos de objeto de estudio, que deben ser considerados por alguna otra teoría. (C, p. 175)

No está claro por qué (8) sería una forma de criticar al veritismo. De hecho, si reemplazamos la variable $x$ con los valores que Kvanvig cree que son problemáticos para el veritismo, mi posición se parece mucho a lo que expresa (8). La utilidad práctica y el placer psicológico son valores tales que creo que es correcto que el veritismo no se ocupe de ellos; sin embargo, es una aspiración legítima pensar que debe haber otras teorías que se ocupen de explicar su naturaleza $-\mathrm{y}$ de hecho sabemos que tales teorías existen-, aunque esas teorías no sean teorías del valor epistémico.

Si pudiera sustancializarse, sería (7) la crítica que representaría un problema grave para el veritismo. Pero la posición de Kvanvig no consigue presentar un caso convincente de (7), pues, como ya señalé, no hay razón para pensar que los valores que él cita sean valores de los que una teoría del valor epistémico debería ocuparse, y sí hay razones sólidas para rechazar que esto sea así. Sin embargo, Cresto parece pensar que inlcuso una posición como la de Kvanvig consigue plantear un problema al veritismo, pues señala que "[si] hay en el entendimiento rasgos que no tienen que ver con la verdad, entonces el veritismo tiene 
un problema, más que una respuesta, para poder afirmar que las pretensiones de los teóricos del entendimiento son ilegítimas" (C, pp. 175176). Nuevamente, la corrección de esta afirmación depende de que haya razón para pensar que los rasgos del entendimiento que están en discusión sean epistémicamente valiosos; el hecho de que sean valiosos de cualquiera de las múltiples formas en las que algo puede ser valioso no basta para convertirlos en explananda legítimos para una teoría del valor epistémico. En F, pp. 165-166, reconozco que hay rasgos asociados con la sistematicidad propia del entendimiento tales que hay razón sólida para pensar que son epistémicamente valiosos; por ejemplo, esa sistematicidad puede promover la explicación y la predicción correctas. Pero insisto en que no cualquier rasgo asociado con la sistematicidad propia del entendimiento que sea valioso de alguna forma constituye ipso facto un explanandum legítimo para una teoría de la evaluación epistémica.

Cualquier cosa es valiosa por muchas razones diferentes; no es sensato pretender que una misma teoría deba dar cuenta de todas las formas en las que algo puede ser valioso. La sistematicidad en un conjunto de creencias es valiosa por razones pragmáticas, psicológicas, epistemológicas y de otro tipo. Suponer que una teoría del valor epistémico debe explicar la naturaleza de todos estos valores heterogéneos sería ignorar las diferencias sustanciales que hay entre ellos y las razones de peso que hay para mantenerlos separados. El placer que la sistematicidad del entendimiento puede producir, la utilidad que a partir de ella podemos ganar para múltiples fines prácticos y la adecuación con la que representa y explica el fenómeno del que se ocupa, son variables independientes, cada una puede moverse independientemente de la otra. A la teoría de la evaluación epistémica le interesa la tercera variable: la relación del entendimiento con el fenómeno del que se ocupa, no la relación del entendimiento con las emociones ni con los fines prácticos del sujeto, pues es aquella variable la que interesa en la empresa de evaluar nuestros intentos por entrar en contacto cognitivo con la realidad.

La importancia de mantener separados los valores involucrados en cada uno de los campos señalados, y de estudiarlos en teorías separadas, se manifiesta en el hecho de que las diferencias que los separan desempeñan un papel central en nuestra forma de dar sentido y entender muchos aspectos de nuestra cultura. Por ejemplo, cuando en un examen universitario evaluamos el entendimiento que un sujeto tiene de un determinado fenómeno, no nos interesan los efectos emocionales que ese entendimiento tenga sobre la vida del examinado, ni cuáles de 
sus problemas prácticos específicos le pueda ayudar a resolver, sino sólo si el entendimiento que ha logrado se relaciona adecuadamente con el fenómeno del que se ocupa. A la inversa, cuando evaluamos los efectos psicológicos del entendimiento sobre la vida del sujeto o los problemas prácticos específicos que de hecho le ayuda a resolver, no nos interesa si su entendimiento se relaciona adecuadamente con el fenómeno del que se ocupa, sino cuáles de sus rasgos hacen que tenga los efectos psicológicos y prácticos que de hecho tiene sobre la vida del sujeto. ${ }^{19}$ Está claro que el sentido de muchas de nuestras prácticas evaluativas presupone que los valores involucrados en los casos descritos son de tipos sustancialmente diferentes que ameritan teorización separada. ${ }^{20}$

La teorización sobre esos diversos valores requiere trazar líneas divisorias que delimiten el campo propio de cada teoría de evaluación. El teórico veritista de la evaluación epistémica circunscribe el campo de la evaluación epistémica con base en su concepción (VE)* del valor epistémico. Cresto tiene razón en que el modo veritista de trazar la línea que separa los valores de los que debe ocuparse una teoría del valor epistémico de aquellos de los que no, no se fundamenta adecuadamente en muchas de las discusiones conocidas. Pero en la primera parte de este trabajo esbocé una manera en la que esa fundamentación puede proceder para que el modo veritista de identificar el domino de la evaluación epistémica, lejos de que sea una mera estipulación idiosincrásica, constituya la base de una teoría explicativamente poderosa y robustamente corroborada de la evaluación epistémica.

\section{BIBLIOGRAFÍA}

Alston, W., 2005, Beyond "Justification". Dimensions of Epistemic Evaluation, Cornell University Press, Ithaca/Londres.

- 1988, "The Deontological Conception of Justification", en J.E. Tomberlin, (comp.), Philosophical Perspectives, vol. 2, Epistemology, pp. 257-299.

${ }^{19}$ Desde luego, el entendimiento puede tener los efectos psicológicos y prácticos que de hecho tiene precisamente gracias a que tiene algún valor epistémico; pero lo que mantiene separado a este valor de los otros es que aquellos pueden presentarse sin éste, no están constitutivamente asociados con él.

${ }^{20} \mathrm{El}$ sentido de muchas discusiones filosóficas clásicas que han sido enormemente fructíferas también presupone las distinciones axiológicas de las que estoy hablando, y trazadas exactamente como las traza el veritista. Por ejemplo, muchas discusiones en filosofía de la ciencia presuponen que hay una distinción entre virtudes pragmáticas y virtudes epistémicas de las teorías científicas, donde la diferencia entre ambas se concibe exactamente como la concebiría el veritista. Una ilustración de esto puede verse en van Fraassen 1980, caps. 2 y 4; véase especialmente la p. 88. 
Brogaard, B., 2009, "The Trivial Argument for Epistemic Value Pluralism, or, How I Learned to Stop Caring about Truth", en A. Haddock, A. Millar, y D.H. Pritchard (comps.), Epistemic Value, Oxford University Press, Oxford, 2009, pp. 285-305.

DePaul, M. y L. Zagzebski (comps.), 2003, Intellectual Virtue: Perspectives from Ethics and Epistemology, Oxford University Press, Oxford.

Feldman, R., 1988, "Epistemic Obligations", en J.E. Tomberlin (comp.), Philosophical Perspectives, vol. 2, Epistemology, pp. 235-256.

Fernández, M.Á., 2010a, “¿Son problemáticos para el veritismo la naturaleza y el valor del entendimiento?”, Diánoia, vol. 55, no. 65, pp. 151-173.

—, $2010 \mathrm{~b}$, "Aptness and Safety: How Are They Related?", Crítica. Revista Hispanoamericana de Filosofía, vol. 42, no. 125, pp. 27-46.

Goldman, A., 1999, Knowledge in a Social World, Oxford University Press, Oxford.

Goodman, N., 1965, Fact Fiction and Forecast, Bobbs-Merrill, Nueva York.

Greco, J., 2003, "Knowledge as Credit for True Belief", en DePaul y Zagzebski 2003, pp. 111-134.

Kvanvig, J., 2003, The Value of Knowledge and the Pursuit of Understanding, Cambridge University Press, Nueva York.

Lycan, W.G., 1986, "Tacit Belief”, en R.J. Bogdan (comp.), Belief. Form, Content and Function, Clarendon Press, Oxford, pp. 61-82.

Pritchard, D., 2010, "The Value Problem for Knowledge", en A. Haddock, A. Millar, y D.H. Pritchard, The Nature and Value of Knowledge: Three Investigations, Oxford University Press, Oxford, pp. 5-25.

_, 2008 , "Sensitivity, Safety and Anti-Luck Epistemology", en John Greco (comp.), The Oxford Handbook of Skepticism, Oxford University Press, Oxford, 2008, pp. 437-455.

Rawls, J., 1971, A Theory of Justice, Harvard University Press, Cambridge.

Riggs, W., 2003, "Understanding Virtue and the Virtue of Understanding", en DePaul y Zagzebski 2003, pp. 203-226.

Sosa, E., 2007, A Virtue Epistemology. Apt Belief and Reflective Knowledge, vol. I, Oxford University Press, Oxford.

—, 2003 , "The Place of Truth in Epistemology", en DePaul y Zagzebski 2003, pp. 155-180.

Van Fraassen, B.C., 1980, The Scientific Image, Clarendon Press, Oxford. [Versión en castellano: La imagen científica, trad. Sergio Martínez, Instituto de Investigaciones Filosóficas-UNAM/Paidós/Seminario de Problemas Científicos y Filosóficos-UnAM, México, 1996.]

Zagzebski, L., 2001, "Recovering Understanding", en M. Steup (comp.), Knowledge, Truth and Duty. Essays on Epistemic Justification, Responsibility and Virtue, Oxford University Press, Oxford, 2001, pp. 235-252.

Recibido el 12 de agosto de 2011; aceptado el 29 de septiembre de 2011. 